\title{
Trends in incidence and tumour thickness of invasive cutaneous melanoma from 2000 to 2014 in an Eastern Hungarian melanoma centre
}

Eszter Anna Janka, Kriszta Kékedi, Emese Gellén, Éva Remenyik, Gabriella Emri Department of Dermatology Faculty of Medicine University of Debrecen, Hungary

\section{Introduction}

\begin{abstract}
The increase in the incidence of malignant melanoma is maintained in the last decades among the fair-skinned population. However, early diagnosis of malignant melanoma can improve prognosis, patient's survival, quality of life and cost savings for health care systems. Primary and secondary prevention programs were initiated in Hungary in the 2000's, but there are only a few studies determining whether the current prevention programs are effective. Epidemiological study on the change in the characteristics of patients and their melanomas can answer this question and might help to develop a more effective prevention strategy.
\end{abstract}

\section{Aims}

Our aim was to assess the incidence, type and localization of melanomas diagnosed at the Department of Dermatology University of Debrecen in the period of 2000-2014. We analyzed the patient's gender and age composition, and the trend in tumour thickness over the examined time period.

\section{Materials and methods}

Source data: malignant melanoma database at the Department of

Dermatology University of Debrecen in Hungary and the MedSolution register database

We processed the data of 1509 primary melanomas. The tumours were categorized according to the American Joint Committee on Cancer 7th edition (2009) TNM staging classification at the time of diagnosis. For the purpose of determining the trend, the data were analyzed for 3-year periods.

\section{Statistical analysis}

The five periods were compared in respect of age and Breslow thickness by ANOVA test, supplemented with Tukey post hoc test. Multinomial logistic regression analysis was used to study the relations between variables in order to determine the change in the clinicopathological parameters over the examined time period. The age-standardized incidence rates of Eastern Hungarian region were calculated by indirect standardization.

\section{Results}

Table 1. Descriptive statistics

\begin{tabular}{|c|c|c|c|c|c|}
\hline Patients & $\begin{array}{c}2000-2002 \\
N=229\end{array}$ & $\begin{array}{c}2003-2005 \\
N=266\end{array}$ & $\begin{array}{c}2006-2008 \\
N=320\end{array}$ & $\begin{array}{c}2009-2011 \\
N=342\end{array}$ & $\begin{array}{c}2012-2014 \\
N=307\end{array}$ \\
\hline Gender N (\%) & 105 (45.9) & $108(40.6)$ & $138(43.1)$ & $156(45.6)$ & $133(43.3)$ \\
\hline Female & $124(54.1)$ & $158(59.4)$ & $182(56.9)$ & $186(54.4)$ & $174(56.7)$ \\
\hline Age (year) mean $\pm S D$ & $56.63 \pm 16.44$ & $56.93 \pm 15.88$ & $57.68 \pm 16.32$ & $59.92 \pm 16.52$ & $59.86 \pm 15.75$ \\
\hline Melanoma & $\mathrm{N}=\mathbf{2 3 1}$ & $N=271$ & $\mathrm{~N}=338$ & $\mathrm{~N}=351$ & $N=318$ \\
\hline Breslow $(\mathrm{mm})$ median & 1.52 & 0.80 & 0.72 & 0.78 & 0.96 \\
\hline AJCC staging $N(\%) \quad I A$ & $67(29.0)$ & 146 (53.9) & $186(55.0)$ & $184(52.4)$ & $151(47.5)$ \\
\hline IB & $22(9.5)$ & $34(12.5)$ & $33(9.8)$ & $32(9.1)$ & $39(12.3)$ \\
\hline$\| \mathrm{A}$ & $27(11.7)$ & $19(7.0)$ & $22(6.5)$ & $30(8.5)$ & $16(5.0)$ \\
\hline IIB & $24(10.4)$ & $15(5.5)$ & $19(5.6)$ & $28(8.0)$ & 30 (9.4) \\
\hline IIC & $11(4.8)$ & $16(5.9)$ & $20(5.9)$ & $18(5.1)$ & 15 (4.7) \\
\hline III & 47 (20.4) & $27(10.0)$ & $30(8.9)$ & $29(8.3)$ & $42(13.2)$ \\
\hline IV & $11(4.8)$ & $7(2.6)$ & $19(5.6)$ & $13(3.7)$ & $22(6.9)$ \\
\hline
\end{tabular}

The most frequent type of melanoma was SSM in all periods. Among females the lower extremities had significantly more frequent occurrence of melanoma, while the trunk was significantly more frequently seen as site of melanoma among men.

Figure 1. Comparing the mean tumour thickness in five periods

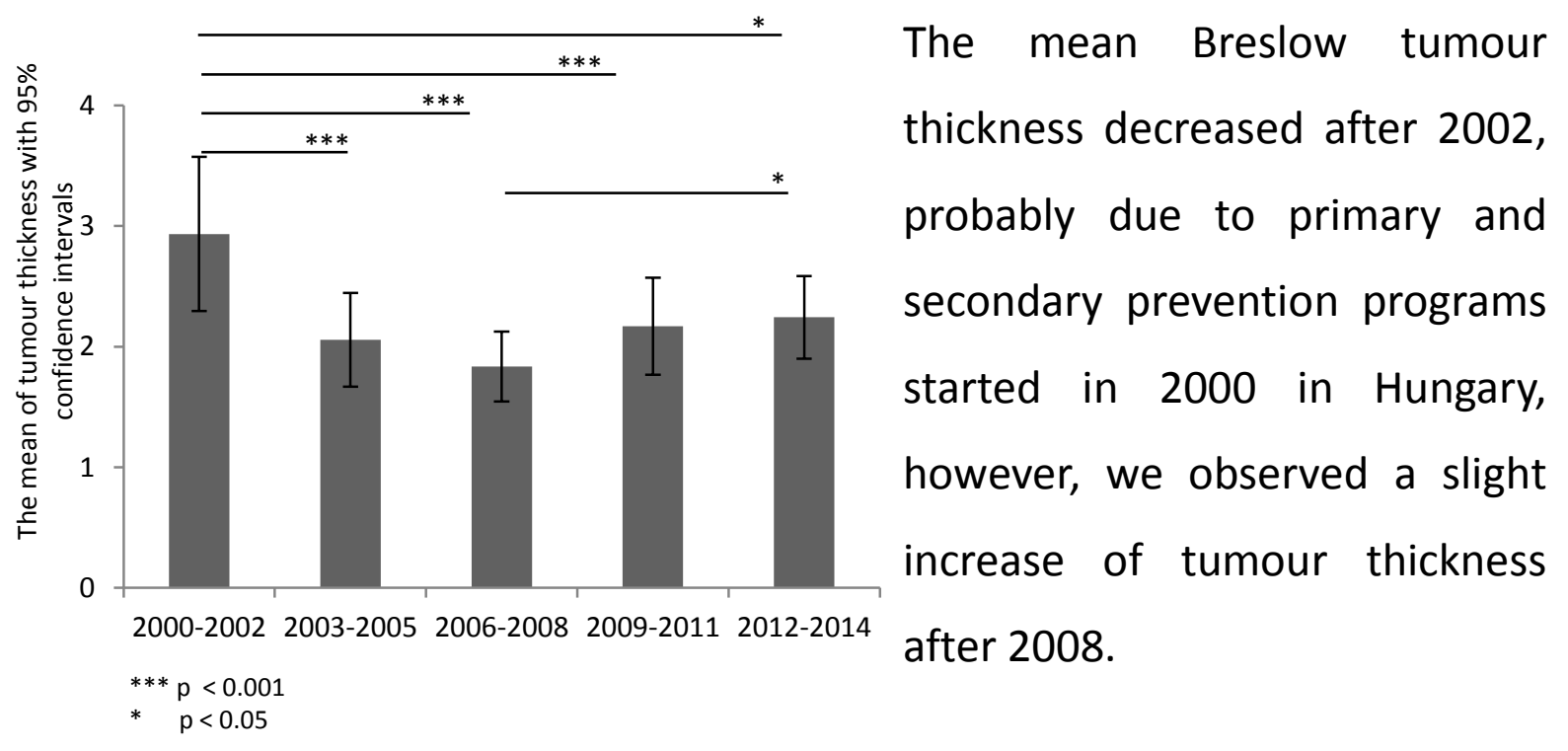

Table 2. The association between Breslow type and variables

\begin{tabular}{|c|c|c|}
\hline Reference: Reference: $\leq 1 \mathrm{~mm}$ thickness & Variables & OR $[95 \% \mathrm{Cl}]$ \\
\hline \multirow{2}{*}{$1.01-2.0 \mathrm{~mm}$} & 2009-2011/2000-2002 & $0.51[0.30 ; 0.88]$ \\
\hline & $2006-2008 / 2000-2002$ & $0.55[0.33 ; 0.94]$ \\
\hline \multirow{7}{*}{$2.01-4.0 \mathrm{~mm}$} & $2009-2011 / 2000-2002$ & $0.40[0.24 ; 0.67]$ \\
\hline & $2006-2008 / 2000-2002$ & $0.31[0.18 ; 0.53]$ \\
\hline & $2003-2005 / 2000-2002$ & $0.38[0.22 ; 0.65]$ \\
\hline & male/female & $1.46[1.03 ; 2.05]$ \\
\hline & $\geq 80 /<40$ & $4.08[1.88 ; 8.84]$ \\
\hline & $60-79 /<40$ & $2.87[1.59 ; 5.19]$ \\
\hline & $40-59 /<40$ & $1.96[1.08 ; 3.57]$ \\
\hline \multirow{8}{*}{$>4 \mathrm{~mm}$} & $2009-2011 / 2000-2002$ & $0.42[0.25 ; 0.72]$ \\
\hline & $2006-2008 / 2000-2002$ & $0.42[0.25 ; 0.73]$ \\
\hline & $2003-2005 / 2000-2002$ & $0.43[0.24 ; 0.75]$ \\
\hline & male/female & $1.42[1.01 ; 2.01]$ \\
\hline & $\geq 80 /<40$ & $7.67[3.92 ; 14.99]$ \\
\hline & $60-79 /<40$ & $2.55[1.48 ; 4.40]$ \\
\hline & trunk/upper extremities & $2.17[1.22 ; 3.85]$ \\
\hline & $\begin{array}{c}\text { lower extremities/upper } \\
\text { extremities }\end{array}$ & $4.69[2.58 ; 8.50]$ \\
\hline
\end{tabular}

The thicker tumours were more likely to appear among men, elder population and on the lower extremities. Furthermore, thicker tumours were significantly less likely to occur in the 2003-2011 period compared to 2000-2002, but in the 2012-2014 period the number of thicker tumours increased again.

Figure 2. The incidence of melanoma among age groups during 2001-2014 periods

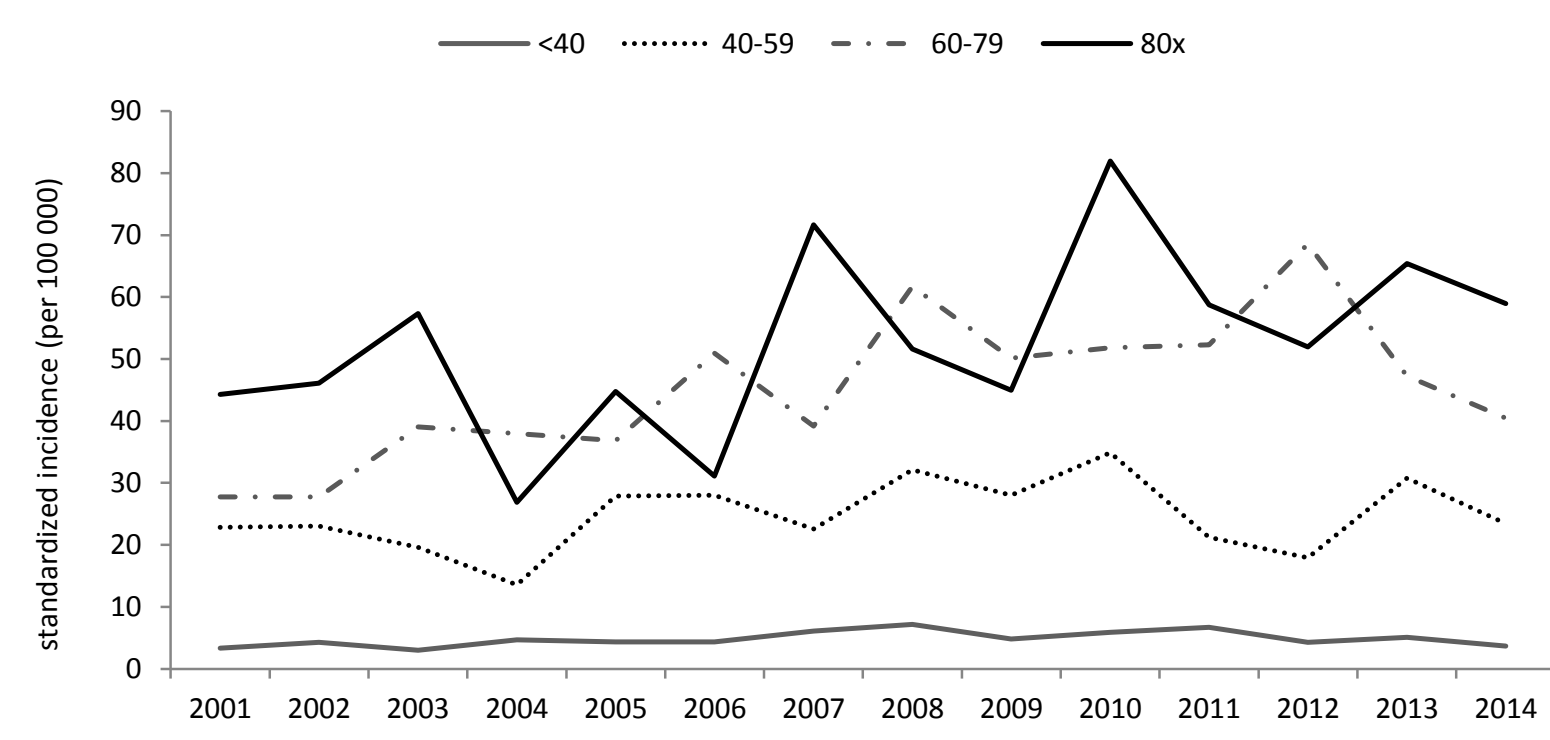

The incidence of melanoma increases with age.

\section{Conclusion}

It seems that primary and secondary prevention programs had beneficial effect on early diagnosis of melanoma in Hungary in the 2000's, however, there is a high need to renew the prevention strategy since we observed higher incidence of melanomas with worse prognosis from 2012. 